\title{
Perception of upper lip augmentation utilizing simulated photography
}

\author{
Gary Linkov, Elizabeth Wick, Dorina Kallogjeri, Collin L. Chen, Gregory H. Branham \\ Department of Otolaryngology-Head \& Neck Surgery, Washington University School of Medicine, St. Louis, MO, USA
}

Background No head to head comparison is available between surgical lip lifting and upper lip filler injections to decide which technique yields the best results in patients. Despite the growing popularity of upper lip augmentation, its effect on societal perceptions of attractiveness, successfulness and overall health in woman is unknown.

Methods Blinded casual observers viewed three versions of independent images of 15 unique patient lower faces for a total of 45 images. Observers rated the attractiveness, perceived success, and perceived overall health for each patient image. Facial perception questions were answered on a visual analog scale from 0 to 100, where higher scores corresponded to more positive responses.

Results Two hundred and seventeen random observers with an average age of 47 years (standard deviation, 15.9) rated the images. The majority of observers were females $(n=183$, $84 \%)$ of white race $(n=174,80 \%)$ and had at least some college education $(n=202,93 \%)$. The marginal mean score for perceived attractiveness from the natural condition was 1.5 points (95\% confidence interval [Cl], 0.9-2.18) higher than perceived attractiveness from the simulated upper lip filler injection condition, and 2.6 points higher $(95 \% \mathrm{Cl}, 1.95-3.24)$ than the simulated upper lip lift condition. There was a moderate to strong correlation between the scores of the same observer.

Conclusions Simulated upper lip augmentation is amenable to social perception analysis. Scores of the same observer for attractiveness, successfulness, and overall health are strongly correlated. Overall, the natural condition had the highest scores in all categories, followed by simulated upper lip filler, and lastly simulated upper lip lift.

Keywords Lip / Surgery, plastic / Injections / Perception
Correspondence: Gary Linkov Department of Otolaryngology-Head \& Neck Surgery, Washington University School of Medicine, $660 \mathrm{~S}$ Euclid Ave, St. Louis, MO 63110, USA Tel: +1-314-362-7509 Fax: +1-314-996-8610 E-mail: glinkov@gmail.com

REDCap supported by Clinical and Translational Science Award (CTSA) Grant (UL1 TR000448) and Siteman Comprehensive Cancer Center and $\mathrm{NCl}$ Cancer Center Support Grant (P30 CA091842).

\section{INTRODUCTION}

According to the 2017 American Society of Plastic Surgeons statistics report, surgical lip augmentation has increased 5\% since 2016 and $60 \%$ since 2000 and soft tissue fillers have increased $312 \%$ since 2000 [1]. We are inundated with photographs of augmented lips in pop culture and patients are pre- senting with specific requests to improve their lip aesthetic. Prior studies have tried to define ideal lip proportions [2-6]. Injectable fillers can three-dimensionally restore the natural contours of the lips and perioral area, thereby reducing some signs of aging lips. The upper lip lift, using various techniques, has been employed by plastic surgeons for over 30 years to reduce the height of the white lip and establish more youthful proportions 
[7-20].

No head to head comparison is available between surgical lip lifting and filler injections to decide which technique yields the best results in patients who meet criteria for surgical correction. Also, despite the growing popularity of upper lip augmentation, its effect on societal perceptions of attractiveness, successfulness and overall health in woman is unknown. These parameters have previously been assessed for rhinoplasty [21] and hair transplant [22].

The aim of the present study was to compare the observer ratings of domains of attractiveness, successfulness, and overall health in women, between three conditions: a simulated filler injection, a simulated surgical lip lift and an original photograph. In addition, we sought to quantify the effect of simulated upper lip augmentation on each of the domains.

\section{METHODS}

\section{Participants}

Institutional Review Board approval (IRB No. 201712019) was received for this study. A total of 217 random observers participated in the study from December 1, 2017, to February 1, 2018. A REDCap (Nashville, TN, USA) produced web-based survey was distributed via the University Volunteer for Health program research participant registry. Observers were asked to provide demographic information, including: age, sex, race, and education level. All individuals 18 years or older who spoke English were eligible. Electronic informed consent was obtained from all study participants, and all data were deidentified. Participants were not compensated. They were naive to the specific study objectives and assumed to be independent.

\section{Instrument}

Photographs of 15 female patients (mean age, 51 years; standard deviation [SD], 6.5 years) who met criteria for surgical upper lip lift (nasal base to lip width ratio $\sim 1: 2$, upper white lip $>18 \mathrm{~mm}$ [23]) and who consented to the use of photographs were included in the study. Patients were selected from a database containing photographs of patients who have been treated within the division of facial plastic and reconstructive surgery. Exclusion criteria for photo use included: heavy make-up, poor lighting, lip lesions or obvious facial scars, mouth open, and prominent perioral rhytids.

Each of the 15 patient photographs went through a series of editing steps using Adobe Photoshop CC 2014 (Adobe Systems, San Jose, CA, USA). The face was cropped down to the bottom half, below the lid/cheek junction, which was done to encourage observer focus on the lips and reduced patient identi- fiers. All photographs were standardized to a right corneal diameter of $11.5 \mathrm{~mm}$.

Photoshop editing was performed on preoperative photographs to create the effect of a $6 \mathrm{~mm}$ reduction of subnasal white lip to represent a typical surgical upper lip lift. Specifically, the transform warp feature was used to shift the upper lip up within the zone of the nasal base to reduce the white lip by $6 \mathrm{~mm}$ and then blending this effect in with the rest of the upper lip. The remaining defect in the upper lip was filled in with the clone stamp tool using various size brushes, followed by the smudge tool to recreate natural results. Editing was also performed to create the effect of upper lip filler injections by using the transform warp feature across the entire upper lip to create a red lip height $1 \mathrm{~mm}$ larger than the original by measuring at the midline and mid nasal sill. This simulated the increase of the Medicis Lip Fullness Scale [24] grade by 1, such as from "very thin" to "thin," or "full" to "very full." A similar effect was seen on the 5 points Allergan Lip Fullness Scale in a recent randomized controlled study assessing the safety and effectiveness of new filler for lip enhancement [25]. In summary, there was one original cropped photograph to show the lower face (natural condition) and two modified versions per patient (simulated upper lip filler and simulated upper lip lift), for a total of 45 images. A representative panel of images for one patient is provided in Fig. 1.

Observers electronically assessed the 45 photographs each in a different random order, blinded to simulated photo treatment. They rated perceived attractiveness, perceived success, and perceived overall health for each patient image. Facial perception questions were answered on a visual analog scale bar from 0 to 100 , where higher scores correspond to more positive responses. Observers rated the attractiveness of the face from 0 (least attractive) to 100 (most attractive). Next, observers rated "How successful do you think this person is?" from 0 (complete failure) to 100 (extremely successful) and "How healthy do you think this person appears?” from 0 (extremely unhealthy) to 100 (extremely healthy).

\section{Statistical analysis}

Analysis was conducted using SAS version 9.4 (SAS Institute Inc., Cary, NC, USA) and IBM SPSS Statistics for Windows version 24.0 (IBM Corp., Armonk, NY, USA). Averages and SDs were reported to describe the distribution of scores for attractiveness, successfulness, and overall health for each of the three patient conditions: natural, simulated upper lip filler, simulated upper lip lift. A mixed model analysis, with observer and photo entered as random effects, was employed to explore the differences between the scores of the three patient conditions evaluated by the same observer. Marginal mean differences and 


\section{Fig. 1. Representative photo panel}

Representative panel of three conditions for one patient, including natural (original, unaltered photograph) (A), simulated filler (B), and simulated upper lip lift (C).
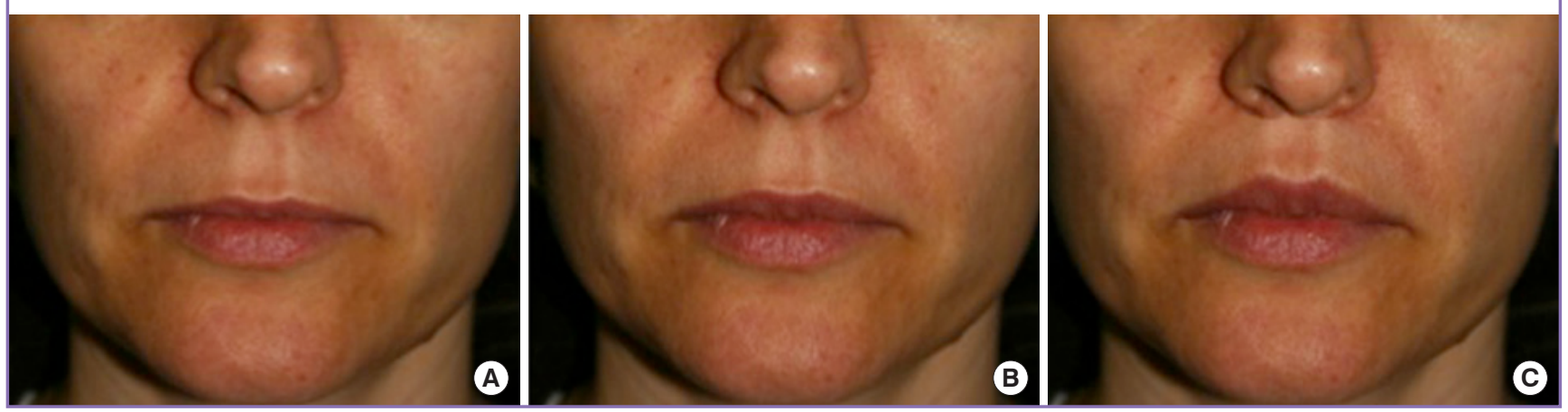

95\% confidence interval ( $95 \% \mathrm{CI})$ around them were used to evaluate magnitude of difference between the scores of the domains and if the $\mathrm{CI}$ included the null value of 0 , the results were deemed statistically nonsignificant. Pearson correlation was used to investigate correlations between the three outcomes measures provided from each observer. Stratified analysis by age of observers categorized as $<50$ and $\geq 50$ was used to explore differences between domain scores for each strata of observers age. In addition, observers gender, race (white vs. other), and education level (categorized in 3 levels) together with ratio of upper to lower lip and age of patients in the photographs were explored as potential confounders in each of the models.

\section{RESULTS}

\section{Description of observers}

Two hundred and seventeen observers rated the images, with an average age of 47 years (SD, 15.9). The majority of observers were females $(n=183,84 \%)$ of white race $(n=174,80 \%)$ and had at least some college education $(n=202,93 \%)$ (Table 1$)$.

\section{Attractiveness}

The overall average attractiveness score for the natural condition was 48.7 (SD, 21.5), for simulated upper lip filler was 47.2 (SD, 21.6); and for simulated upper lip lift was 46.2 (SD, 21.4) (Fig. 2). The marginal mean score for perceived attractiveness from the natural condition was 1.5 points ( $95 \% \mathrm{CI}, 0.9$ to 2.18 ) higher than perceived attractiveness from simulated upper lip filler, and 2.6 points higher ( $95 \% \mathrm{CI}, 1.95$ to 3.24 ) than the simulated upper lip lift. The marginal mean score for perceived attractiveness from simulated upper lip filler was 1.06 points higher (95\% CI, 0.41 to 1.70$)$ than the perceived attractiveness from simulated upper lip lift (Fig. 3). The comparison of average perceived attractiveness scores of the three conditions for each pa-
Table 1. Demographic characteristics of study observers

\begin{tabular}{|lc|}
\hline Observer demographic & Value $(\mathbf{n}=\mathbf{2 1 7})$ \\
\hline Age $(\mathrm{yr})$ & $47.4 \pm 15.9$ \\
Sex & \\
$\quad$ Female & $183(84)$ \\
$\quad$ Male & $34(16)$ \\
Race/ethnicity & \\
$\quad$ White & $174(80)$ \\
Asian & $4(2)$ \\
Hispanic & $4(2)$ \\
African American & $32(15)$ \\
Other & $3(1)$ \\
Educational status & \\
<High school & $1(1)$ \\
High school & $14(7)$ \\
Some college & $35(16)$ \\
2-Year college degree & $19(9)$ \\
4-Year college degree & $75(35)$ \\
Master's degree & $56(26)$ \\
Doctoral degree & $17(8)$ \\
\hline Values are presented as mean \pm SD or number (\%). & \\
\hline
\end{tabular}

tient photo is displayed in Fig. 4A.

\section{Successfulness}

The overall average successfulness score for natural condition was 54.5 (SD, 19.7), for simulated upper lip filler was 54.0 (SD, 19.6); and for simulated upper lip lift was 52.4 (SD, 19.8) (Fig. 2). The marginal mean score for perceived successfulness from the natural condition was 0.4 points ( $95 \% \mathrm{CI},-0.20$ to 1.01 ) higher than perceived successfulness from simulated upper lip filler, and 2.1 points higher (95\% CI, 1.52 to 2.74 ) than the simulated upper lip lift. The marginal mean score for perceived successfulness from simulated upper lip filler was 1.7 points higher ( $95 \% \mathrm{CI}, 1.11$ to 2.33 ) than the perceived successfulness from simulated upper lip lift (Fig. 3). The comparison of average per- 


\section{Fig. 2. Grouped scores by survey respondents}

Box plots of scores assigned by survey respondents evaluating photos of women for perceived attractiveness, health, and level of success. (A) Scores visually grouped by type of personal attribute. (B) Scores visually grouped by type of simulated treatment. CP, crude photo (natural); SLL, simulated upper lip lift; SLF, simulated upper lip filler; $A$, attractive; $H$, healthy; $S$, successful.
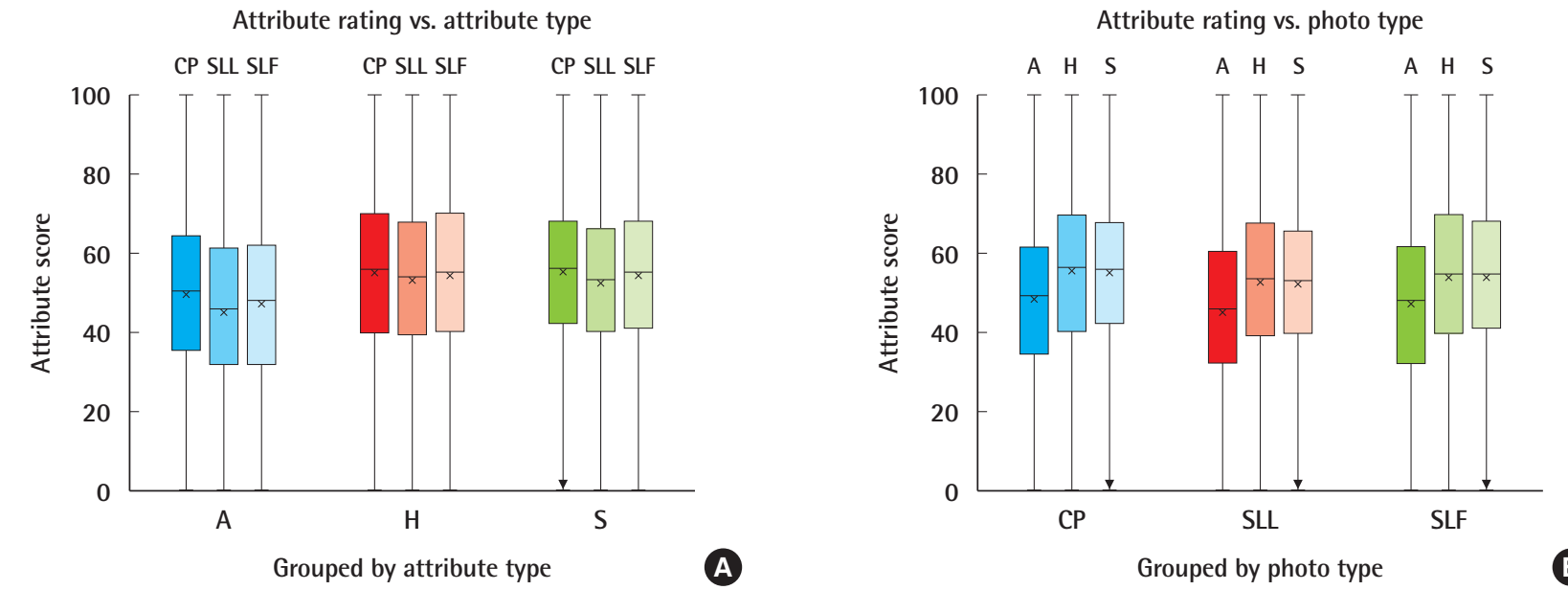

\section{Fig. 3. Marginal mean score of various treatments}

Marginal differences between scores assigned to different treatments. The 95\% confidence intervals (CI) around mean marginal differences for scores of attractiveness, health, and success assigned to each of the three photo types including the crude photo (CP), simulated upper lip lift (SLL), and simulated upper lip filler (SLF). The horizontal axes have been reduced in scale to improve visualization of the statistically significant marginal differences. Positive values indicate that the treatment listed first (left vertical axis) received a higher score in each of the nine clusters. Statistical significance indicated by all parameters that do not include zero marginal mean score.
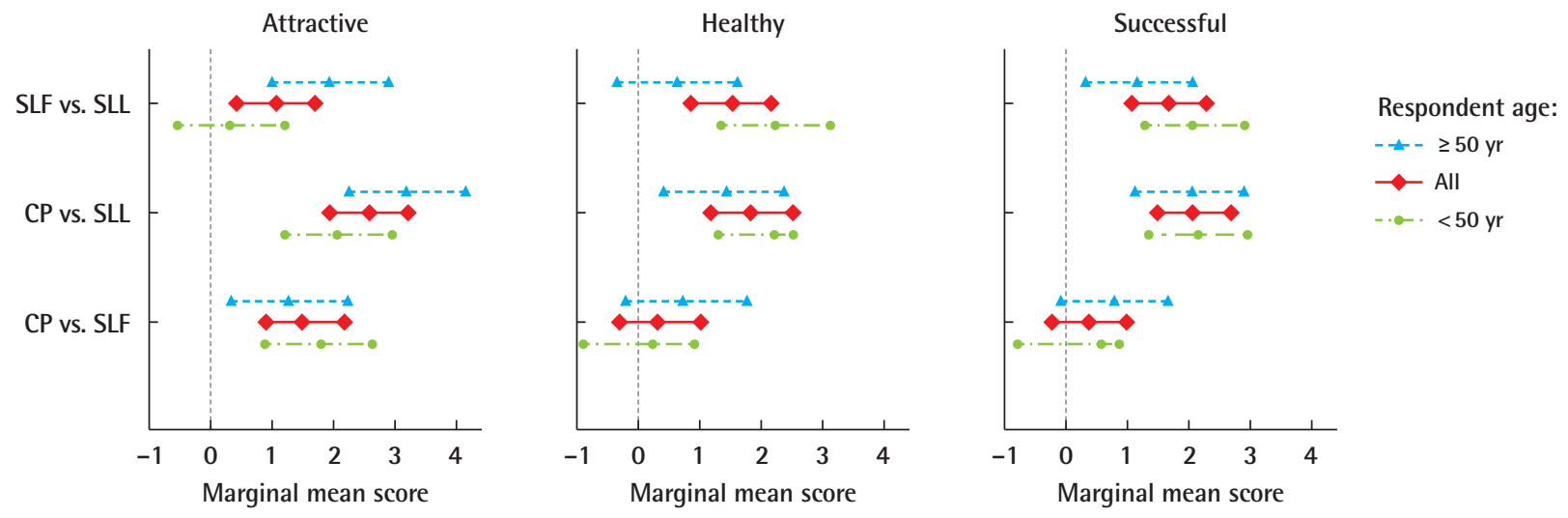

ceived successfulness scores of the three conditions for each patient photo is displayed in Fig. 4B.

\section{Overall health}

The average overall health score for natural condition was 54.9 (SD, 21.6), for simulated upper lip filler was 54.5 (SD, 21.8); and for simulated upper lip lift was 53.1 (SD, 21.5) (Fig. 2). The mixed model analysis showed that marginal mean score for perceived overall health from the natural condition was 0.3 points (95\% CI, -0.33 to 0.99 ) higher than perceived overall health from simulated upper lip filler, and 1.8 points higher (95\% CI, 1.15 to 2.48 ) than the simulated upper lip lift. The marginal mean score for perceived overall health from simulated upper lip filler was 1.5 points higher ( $95 \% \mathrm{CI}, 0.82$ to 2.14 ) than the perceived overall health from simulated upper lip lift (Fig. 3). The comparison of average perceived overall health scores of the three conditions for each patient photo is displayed in Fig. 4C.

\section{Pearson correlation}

Distribution of Pearson correlation coefficients for the relation- 


\section{Fig. 4. Perception scores across conditions}

Perception scores across conditions for each patient. (A) Attractiveness, (B) successfulness, (C) overall health. 1, crude (natural); 2, simulated filler; 3, simulated upper lip lift.
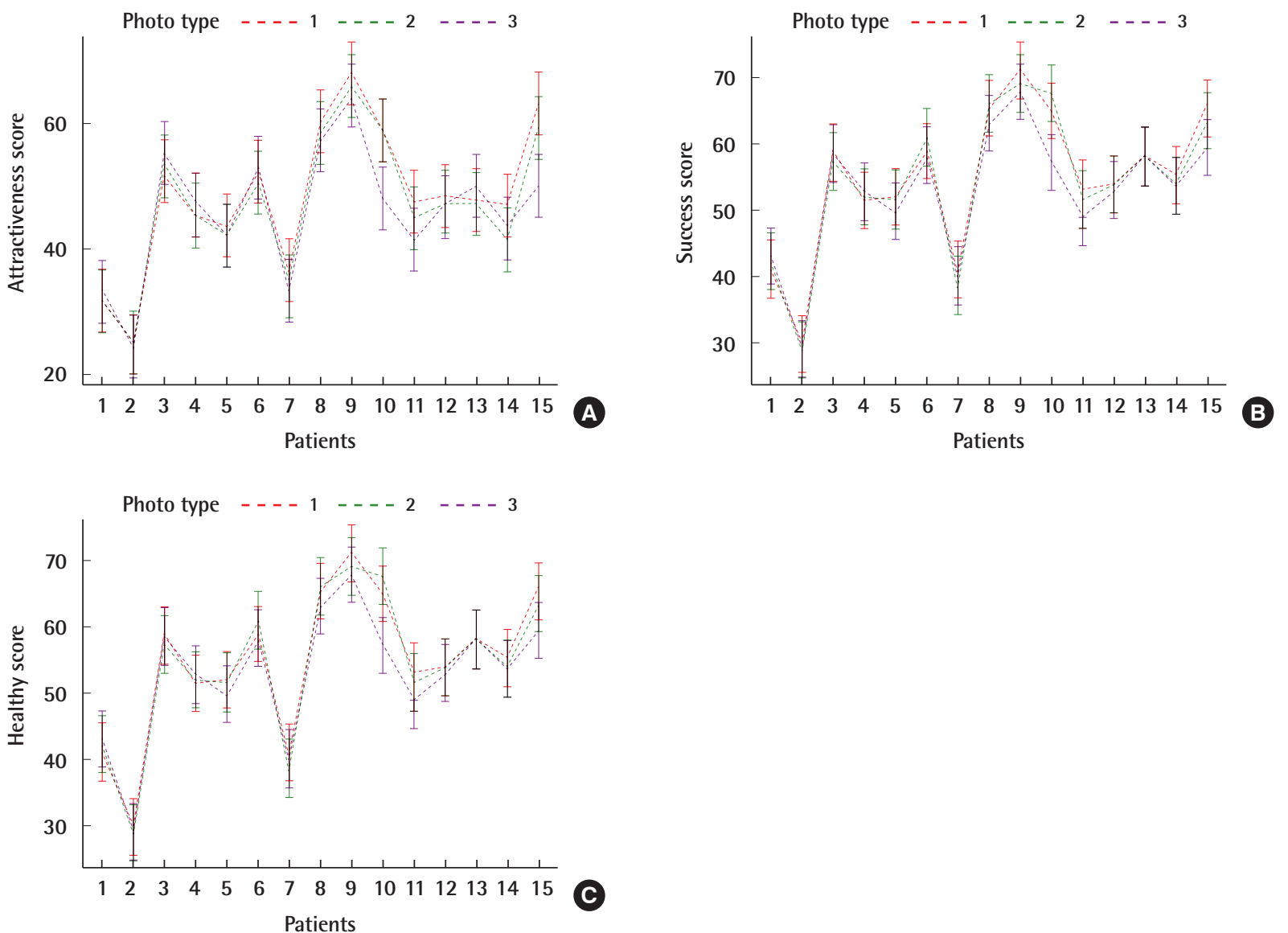

ship between attractiveness and overall health per each observer had a median of 0.78 and interquartile range (IQR; $0.66-0.87$ ). The distribution of Pearson correlation coefficients for the relationship between attractiveness and successfulness per each observer had a median of 0.79 and IQR (0.62-0.90). The distribution of Pearson correlation coefficients for the relationship between overall health and successfulness per each observer had a median of 0.81 and IQR $(0.65-0.90)$.

\section{DISCUSSION}

In this study, naïve observers blinded to patient simulated upper lip augmentation status (natural, filler, and lift) rated independent images of patients on attractiveness, successfulness, and overall health. To date, this is the only study that we know of that assesses social perception of various lip augmentation strategies, whether by simulation or actual results.

Interestingly, we found that for all three of the social percep- tion measures, the unaltered, natural image had higher ratings than both the filler and lift versions. The filler version was more favorable than the lift. These results were statistically significant but within a relatively narrow range of values, similar to other social perception studies in facial plastic surgery $[21,22]$.

Why would patients with greater enhancement of the upper lip and shortening of the upper cutaneous lip be seen as less attractive, less successful, and in poorer overall health? Why is lip augmentation becoming more popular if social perception would suggest that a more natural appearance is superior?

While there may be many plausible explanations, one clue could be found in a 2017 manuscript by Popenko et al. [2]. Using a robust sample size with digitally manipulated images and focus group raters, they found that the most attractive upper to lower lip ratio is $1: 2$ (compared to $1: 1,1: 3,2: 1$ ). Overall, these findings were consistent with the ratio of most natural lips before any augmentation procedure. They conclude by advocating for preservation of the natural ratio or achieving a 1:2 ratio in lip augmenta- 
tion procedures while avoiding the overfilled upper lip look frequently seen among celebrities. Of note, there are different aesthetic standards around the world with variable ideal ratios.

Another study on the attractive lip, by Penna et al. [3], assessed certain parameters of the lip and lower third of the face. While no exact ratio was provided, their results on 88 women and 250 observers, was that female attractiveness is bolstered by a greater upper vermillion height (dry red lip) as well as a greater lower vermillion height. Yet another study looking at the parameters of attractive lips [4] found that the sets of lips rated as most attractive shared five similar parameters that were smaller than average for their sex-based ethnic group, including: upper lip surface midline height, bilateral paramedian lip surface height, upper lip angle, and lower lip volume. Most parameters translated into a relatively thinner upper lip, a stark contrast to the current trend of enhancing upper lip volume.

It would appear that maintaining appropriate proportions of upper to lower red lip is more important than the simple reduction of upper white lip height. We included the ratio of upper to lower red lip in our mixed model analysis to control for their confounding effect. In our study, as we accentuated the upper lip first with a simulated filler version and then with a more pronounced upper lip lift version, the ideal ratio became more distorted and therefore the patient was perceived as less attractive, successful and healthy. Ideal lip augmentation, whether by filler or surgical lip lift, must take into consideration the lower red lip and its proportion to the modified upper lip. A surgical upper lip lift has greater potential than filler to exaggerate white and red lip relationships and therefore must be practiced with caution. Patients who obtain an upper lip lift or filler should be counseled on the potential importance of lower lip augmentation, whether surgical or not, to restore the ideal proportions.

Our study suggests a strong correlation between the various social perception parameters (attractiveness, success, and overall health) per patient photo, suggesting that the nuances of each parameter are less important than the observer's gestalt impression. Furthermore, the most significant rating differences were seen between patients, which highlights that individual patient differences are stronger predictors of outcome than either the observer or the type of augmentation applied. This notion speaks to one of the limitations of our study which is that we did not control for skin tone, degree of rhytids, degree of tissue descent or deflation, proportions of lips to nose and/or lower face. All of these variables likely impact observer ratings.

Another limitation of our study is the use of simulated imaging rather than actual procedure results. Simulations have flaws in the way images are rendered and perhaps perceived. However, actual lip filler and lip lift results will also vary based on patient variability, surgeon expertise, and surgical technique. Whether by simulation or actual procedural intervention, results cannot be generalized to all potential patient outcomes. In addition, a large proportion of our observers are educated, white women from the Midwest, which was the product of conducting a webbased survey and recruiting through the university's research volunteer office.

Despite these limitations, the data suggests that patients with a more natural upper lip are perceived as more attractive, more successful, and overall healthier. Upper lip alterations are possible but should take the upper to lower lip proportions into consideration. Future investigations should assess additional social perception domains using preoperative and postoperative photos of patients from multiple demographics that underwent actual lip filler and lip lift procedures.

\section{NOTES}

\section{Conflict of interest}

No potential conflict of interest relevant to this article was reported.

\section{Ethical approval}

The study was approved by the Institutional Review Board of Washington University School of Medicine (IRB No. 201712019) and performed in accordance with the principles of the Declaration of Helsinki. Written informed consents were obtained.

\section{Patient consent}

The patients provided written informed consent for the publication and the use of their images.

\section{Author contribution}

Conception and design: Linkov G, Kallogjeri D. Analysis and interpretation: Linkov G, Wick E, Kallogjeri D, Chen CL, Branham GH. Manuscript drafting and review: Linkov G, Wick E, Kallogjeri D, Chen CL, Branham GH.

\section{ORCID}

Gary Linkov https://orcid.org/0000-0001-8584-5851

\section{REFERENCES}

1. American Society of Plastic Surgeons. 2017 Plastic surgery statistics report [Internet]. Arlington Heights, IL: American Society of Plastic Surgeons; c2018 [cited 2019 May 3]. Available from https://www.plasticsurgery.org/documents/ 
News/Statistics/2017/plastic-surgery-statistics-report-2017. pdf.

2. Popenko NA, Tripathi PB, Devcic Z, et al. A quantitative approach to determining the ideal female lip aesthetic and its effect on facial attractiveness. JAMA Facial Plast Surg 2017; 19:261-7.

3. Penna V, Fricke A, Iblher N, et al. The attractive lip: a photomorphometric analysis. J Plast Reconstr Aesthet Surg 2015; 68:920-9.

4. Wong WW, Davis DG, Camp MC, et al. Contribution of lip proportions to facial aesthetics in different ethnicities: a three-dimensional analysis. J Plast Reconstr Aesthet Surg 2010;63:2032-9.

5. Sawyer AR, See M, Nduka C. 3D stereophotogrammetry quantitative lip analysis. Aesthetic Plast Surg 2009;33:497504.

6. Haworth RD. Customizing perioral enhancement to obtain ideal lip aesthetics: combining both lip voluming and reshaping procedures by means of an algorithmic approach. Plast Reconstr Surg 2004;113:2182-93.

7. Mommaerts MY, Blythe JN. Rejuvenation of the ageing upper lip and nose with suspension lifting. J Craniomaxillofac Surg 2016;44:1123-5.

8. Lee DE, Hur SW, Lee JH, et al. Central lip lift as aesthetic and physiognomic plastic surgery: the effect on lower facial profile. Aesthet Surg J 2015;35:698-707.

9. Moragas JS, Vercruysse HJ, Mommaerts MY. "Non-filling” procedures for lip augmentation: a systematic review of contemporary techniques and their outcomes. J Craniomaxillofac Surg 2014;42:943-52.

10. Ponsky D, Guyuron B. Comprehensive surgical aesthetic enhancement and rejuvenation of the perioral region. Aesthet Surg J 2011;31:382-91.

11. Echo A, Momoh AO, Yuksel E. The no-scar lip-lift: upper lip suspension technique. Aesthetic Plast Surg 2011;35:617-23.
12. Weston GW, Poindexter BD, Sigal RK, et al. Lifting lips: 28 years of experience using the direct excision approach to rejuvenating the aging mouth. Aesthet Surg J 2009;29:83-6.

13. Waldman SR. The subnasal lift. Facial Plast Surg Clin North Am 2007; 15:513-6.

14. Santanche P, Bonarrigo C. Lifting of the upper lip: personal technique. Plast Reconstr Surg 2004;113:1828-35.

15. Niechajev I. Lip enhancement: surgical alternatives and histologic aspects. Plast Reconstr Surg 2000;105:1173-83.

16. Guerrissi JO. Surgical treatment of the senile upper lip. Plast Reconstr Surg 2000;106:938-40.

17. Jeter TS, Nishioka GJ. The lip lift: an alternative corrective procedure for latrogenic vertical maxillary deficiency: report of a case. J Oral Maxillofac Surg 1988;46:323-5.

18. Austin HW. The lip lift. Plast Reconstr Surg 1986;77:990-4.

19. Fanous N. Correction of thin lips: "lip lift”. Plast Reconstr Surg 1984;74:33-41.

20. Rozner L, Isaacs GW. Lip lifting. Br J Plast Surg 1981;34: 481-4.

21. Nellis JC, Ishii M, Bater KL, et al. Association of rhinoplasty with perceived attractiveness, success, and overall health. JAMA Facial Plast Surg 2018;20:97-102.

22. Bater KL, Ishii M, Joseph A, et al. Perception of hair transplant for androgenetic alopecia. JAMA Facial Plast Surg 2016;18:413-8.

23. Perenack J. Treatment options to optimize display of anterior dental esthetics in the patient with the aged lip. J Oral Maxillofac Surg 2005;63:1634-41.

24. Kane MA, Lorenc ZP, Lin X, et al. Validation of a lip fullness scale for assessment of lip augmentation. Plast Reconstr Surg 2012;129:822e-828e.

25. Geronemus RG, Bank DE, Hardas B, et al. Safety and effectiveness of VYC-15L, a hyaluronic acid filler for lip and perioral enhancement: one-year results from a randomized, controlled study. Dermatol Surg 2017;43:396-404. 